\title{
Adverse Events after Uterine Compression Sutures for Postpartum Hemorrhage: Report of Three Cases and Review of the Literature
}

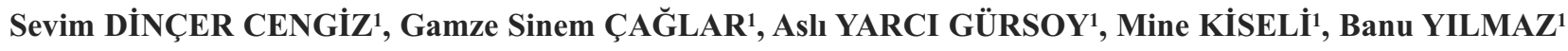 \\ Ankara, Turkey
}

\begin{abstract}
Uterine compression sutures may be life and fertility saving interventions in management of uterine atony. However, unintended long-term outcomes may occur and there is scarce literature about management. Herein, management of long-term adverse outcomes (synechia, pelvic pain and pelvic collection) of three different cases who had to be treated by uterine compression sutures is reported with review of the literature.
\end{abstract}

Keywords: Uterine atony, Adverse outcome, Compression suture

Gynecol Obstet Reprod Med 2016;22:49-53

\section{Introduction}

Postpartum haemorrhage is one of the most common cause of maternal morbidity and mortality worldwide. ${ }^{1}$ When the hemorrhage is due to uterine atony, mechanical and pharmacologic interventions must be performed to stop the bleeding. The management steps of uterine atony can be classified as medical, non-medical and surgical interventions. Medical intervention as first step is composed of uterotonic agents (oxytocin, ergometrine, carbetocin and misoprostol). Non-medical step includes uterine massage, bimanual uterine compression, intrauterine balloon or condom tamponade if available, external aortic compression, uterine artery embolizaton if possible. ${ }^{2}$ The surgical interventions can be listed as uterine compression sutures (UCS), uterine artery ligation, utero-ovarian artery ligation and hypogastric artery ligation. Hysterectomy is the last measure to avoid maternal death. ${ }^{1}$

Among the surgical interventions, UCS are particularly useful and easier to perform when used as uterine sparing procedure to control severe postpartum hemorrhage. Since BLynch suture was introduced in $1997,{ }^{3}$ numerous UCS have been described [Cho multiple square, Hayman, Pereira, Hackethal (interrupted U sutures)].,5 There is no 'the best' suture yet and the success rate of compression sutures varies between $76-100 \%$ in the literature. ${ }^{5}$ Lack of standardization of

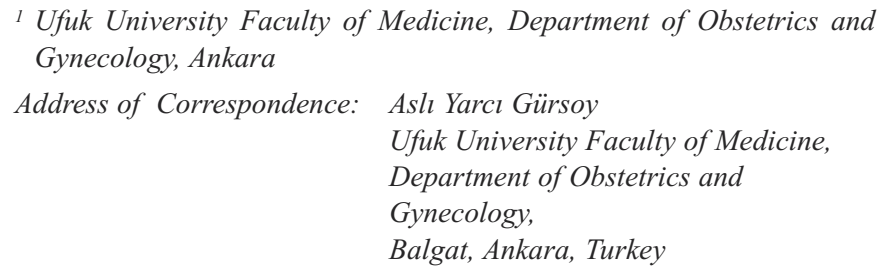

Submitted for Publication:

10. 11. 2014

Accepted for Publication: the procedures and the availability of only case series and stepwise uterine measures make it impossible to efficiently compare the efficacy of different UCS techniques.

Although, UCS are adopted promptly throughout the world, the possible midterm and long-term potential risks related to the procedure are not very clear. The fertility rate after UCS range between 10 to $100 \%$ in the literature with lower percentiles for Hayman's and modified B-Lynch sutures. ${ }^{6}$ Even if, the fertility outcome after UCS is encouraging and no report of infertility induced by the insertion of UCS exists; ${ }^{6}$ pyometra, endometritis, partial uterine necrosis, intrauterine synechia and uterine rupture in the subsequent pregnancy has been reported in long-term. ${ }^{7,8}$ In this report the long-term possible adverse outcomes after UCS are discussed regarding the technique and the suture material used where the recent suggestions in the literature to avoid these problems are mentioned. Moreover, three different clinical occasions (pelvic abscess, intrauterine synechia and pelvic pain) after UCS are also presented (Table 1).

\section{Case Presentation 1: Synechia}

31 year-old patient at her first gestation was admitted to the hospital with amniotic membrane rupture at 38 weeks of pregnancy. At the time of admission, bimanual examination revealed four $\mathrm{cm}$ dilatation accompanied by $60 \%$ effacement with spontaneous amnion leakage. During follow-up of the patient, active phase of delivery was prolonged and oxytocin infusion was started. After four hours, the patient delivered a healthy fetus. Immediately after the delivery of the fetus severe postpartum hemorrhage started. Upon the unresponsiveness to uterine massage, bimanuel uterine compression and uterotonic medications laparatomy was performed. Bakri balloon application or uterine artery embolisation was not feasible. As first line surgical approach uterine artery ligation was 
Table 1: Characteristics of three cases

\begin{tabular}{|l|l|l|l|l|l|l|l|}
\hline & $\begin{array}{l}\text { NVD or } \\
\text { C/S }\end{array}$ & $\begin{array}{l}\text { Uterine Artery } \\
\text { Ligation }\end{array}$ & UCS & $\begin{array}{l}\text { Suture } \\
\text { material }\end{array}$ & $\begin{array}{l}\text { Adverse } \\
\text { Outcome }\end{array}$ & Diagnosis & Intervention \\
\hline Case 1 & NVD & Yes & $\begin{array}{l}\text { U sutures* } \\
(n=8)\end{array}$ & $\begin{array}{l}\text { Polypropylene } \\
\text { USP 1 }\end{array}$ & Uterine synechia & $\begin{array}{l}\text { Hysterosalphingo } \\
\text { graphy }\end{array}$ & Hysteroscopy \\
\hline Case2 & NVD & Yes & $\begin{array}{l}\text { U Sutures* } \\
(n=7)\end{array}$ & $\begin{array}{l}\text { Polypropylene } \\
\text { USP 1 }\end{array}$ & Pelvic pain & $\begin{array}{l}\text { Diagnostic } \\
\text { laparoscopy }\end{array}$ & Laparoscopy \\
Case 3 sutures* & C/S & Yes & $\begin{array}{l}\text { Polypropylene } \\
\text { USP 1 }\end{array}$ & $\begin{array}{l}\text { DIC } \\
\text { Pelvic collection }\end{array}$ & $\begin{array}{l}\text { Ultrasonography } \\
\text { Magnetic } \\
\text { Resonance Imaging }\end{array}$ & Percutaneous drainage \\
\hline
\end{tabular}

*Technically similar with Hackethal suture, changing in number according to the case

UCS: Uterine compression suture, DIC: Disseminated intravascular coagulation

done bilaterally. Since the bleeding did not cease, multiple uterine compression sutures-totally 8-by Hackethal technique were performed. ${ }^{4}$ Polypropylene USP 1 (Prolene ${ }^{\circledR}$, Ethicon, USA) suture was used to cross through anterior and posterior walls of the uterus opposing two walls of the uterus which finally restored hemostasis. The patient was discharged at postoperative day four. The follow-up was uneventful until she stopped breast-feeding at 28 months after delivery. The patient was admitted to hospital with secondary amenorrhea. The hysterosalpingography revealed uterine adhesions. Operative hysteroscopy with the guidance of ultrasonography was performed. Intraoperatively, dense and filmy adhesions in the uterine cavity were relieved by knife electrocautery and the uterine cavity was totally restored. Postoperatively estrogen and progesterone replacement therapy was given which resulted with regular menstrual periods two months later.

\section{Discussion}

Synechia is one of the most common complications reported after UCS. This entity is closely related with future fertility and long term success of the organ preserving surgery. Among the current UCS techniques, except B-lynch, most of the sutures surpass the uterine cavity resulting with increased risk of intrauterine synechia. There is not any controlled trial comparing different techniques but only case reports. But hypothetically B-Lynch suture, not transversing the uterine cavity, may avoid foreign body reaction which may be the leading reason for synechia.

In a recent review, $15 \%(7 / 46)$ of the cases were found to have intrauterine synechia to some extent after UCS. Among these cases five were after Cho technique, one was after combined B-Lynch plus Cho techniques and one was after BLynch. ${ }^{8}$ In this case series the time of diagnosis of synechia ranged from 3 months to 2 years after the procedure. According to this report although there are very few cases, the extent of the synechia seems to increase as the time between the procedure and evaluation increases. According to another report, in patients with UCS performed after cesarean section,
$53 \%(7 / 13)$ were found to have synechia when re-evaluated with hysteroscopy in long-term follow up. The authors proposed that even if UCS is an easier technique when compared with vascular ligation, the long-term fertility outcomes may not be so encouraging. ${ }^{9}$ Other than case reports, a retrospective study from France reported uterine synechia among women after UCS for postpartum hemorrhage. ${ }^{10}$ The integrity of the uterine cavity was confirmed by hysteroscopy or hysterosalpingogram after a median time of 9.3 months (range: 2.4-34.8 months) after delivery. In these cases several transfixing sutures with an atraumatic polyglactin 910 (Vicryl ${ }^{\circledR}$, Ethicon, USA) USP 1 suture were applied to decrease the uterine size and to stop bleeding. Uterine synechiae was found in $26.7 \%(4 / 15)$ of the cases all of whom underwent uncomplicated hysteroscopic resection of the adhesions. ${ }^{10}$

One possible question is whether the type of the suture material used is related to the extent and degree of the synechia. The original technique was defined using a large Mayo needle with chromic catgut suture USP 2 by B-Lynch. ${ }^{3}$ In reports of cases complicated with synechia after UCS, very few authors mentioned about the suture material they used. Among the case reports with synechia after UCS, chromic catgut was used in one patient, ${ }^{11}$ polyglycolic acid in another patient ${ }^{12}$ and atraumatic polyglactin 910 USP 1 was used in four cases. ${ }^{10}$ Since chromic catgut is not available in the market now, long lasting absorbable sutures (either polyglycolic acid USP 0 or 1) are mostly used in the above mentioned case series where $26.7 \%$ $53 \%$ of the patients had synechia after UCS. ${ }^{9,10}$

The optimal time and method for the evaluation of the patients with UCS in order to diagnose and treat synechia is also obscure since the number of the reported cases is very scarce. During follow-up of patients, the possibility of this complication should be kept in mind and the integrity of uterine cavity would rather be evaluated either by hysteroscopy, hysterosalpingography or sonohysterography in the postpartum period where possible. Since early diagnosis seems to be associated with the extent of synechia, we suggest a routine diagnostic and therapeutic hysteroscopy after a short interval from the 
initial procedure. Additionally, B-Lynch if performed by polyglytone 6211 (absorbtion time: 56 days) might contribute to avoid long-term extensive adhesions and to ensure a functional genital tract enabling fertility.

\section{Case Presentation 2: Pelvic Pain/ Dysmenorrhea}

31 year-old primipara patient at 39 weeks of gestation was hospitalized with periodic uterine contractions. The follow-up was uneventful until the second stage of vaginal delivery which lasted about 1.5 hours. Immediately after delivery of the baby, massive bleeding started because of uterine atony. As uterotonic agents and non-medical interventions such as uterine massage and compression were not enough for achieving hemostasis, and other non-surgical interventions (Bakri balloon and embolisation) were not feasible, laparotomy had to be performed. Following bilateral uterine artery ligation, the hemorrhage continued and eight U-sutures were placed with nonabsorbable polypropylene (Prolene ${ }^{\circledR}$, Ethicon, USA) USP 1 suture material starting at the fundus and ending at the lower uterine segment, under manual compression to achieve optimal reduction of the uterine volume until hemostasis was restored. ${ }^{8}$ The patient was discharged after one week. After two months of delivery the patient was admitted to the hospital with severe pelvic pain. Laboratory tests and ultrasonography were all in normal range. A diagnostic laparoscopy was performed. Intraoperatively; previously applied eight sutures through the anterior and posterior sides of the uterus were visible and were all removed with success. Additionally office hysteroscopy was performed which confirmed normal uterine cavity. After removal of the sutures by laparoscopy, pelvic pain ceased. The patient was discharged at postoperative day one.

\section{Discussion}

Pelvic pain usually occurs after UCS. At this point differential diagnosis is required for possible complications. In cases in whom uterine cavity is bypassed by UCS, blood clot and debris entrapment is theoretically possible leading to collection of intrauterine blood (hematometra) or pus (pyometra). ${ }^{8,13,14}$ Also partial or total necrosis of the uterine wall caused by extremely tight sutures may be another cause of pelvic pain. ${ }^{15,16}$ Other than the causes listed above chronic pelvic pain may be due to non-absorbable suture materials used for UCS, as in the case we presented here. For the differential diagnosis of pelvic pain, radiological evaluation by ultrasonography and magnetic resonance imaging may be valuable. Magnetic resonance angiography may be the best option to make differential diagnosis for a necrotic myometrium. ${ }^{15}$

The management and differential diagnosis of a case suffering from pelvic pain with a previous history of uterine atony and surgery can be quite hard if one does not know about the details of the previous surgical procedure. Usually, the patients do not apply to the same medical center where the initial surgical management is performed. ${ }^{17}$ In such a case, the procedure itself, the suture materials used or accompanying vascular interventions (uterine artery ligation, internal iliac artery ligation) of the previous surgery would rather be ascertained. When laboratory and radiological examinations are not adequate to explain the cause of pelvic pain, performing laparoscopy will help to explore the abdominal cavity and an accurate diagnosis will be achieved. The details of the medical story and surgery of the case presented here could not obtained, therefore a diagnostic laparoscopy is required. Finally, we infer that using absorbable suture materials for uterine compression sutures might decrease the necessity for a second look laparoscopy to remove the previously applied sutures in order to relieve pain.

\section{Case Presentation 3: Pelvic Collection}

36 year-old primigravid patient at 39 weeks of gestation admitted to the hospital with regular uterine contractions accompanied by cervical dilatation. Ultrasonography revealed multiple uterine leiomyomas, largest being $7 \times 8 \mathrm{~cm}$ and smallest $2 \times 3 \mathrm{~cm}$ in diameter, and her history revealed previous myomectomy. The delivery was planned by cesarean section. Intraoperatively multiple leiomyomas which were mostly subserous and intramural in origin were observed distributed widely around the uterus. Following the delivery of the fetus, uterine atony developed that did not respond to the compression or uterine massage followed by uterotonic medications. Considering the young age and low parity of the patient, first of all bilateral uterine artery ligation was performed and since hemostasis of the patient was deteriorating through disseminated intravascular coagulation additional eritrocyte suspensions, fresh frozen plasma and trombocyte suspensions were administered intraoperatively. To restore the hemostasis eight $\mathrm{U}$ sutures by non-absorbable polypropylene (Prolene ${ }^{\circledR}$, Ethicon, USA) USP 1 suture were applied crossing through both walls of the uterus until surgical homeostasis was achieved. Since excessive bleeding continued and laboratory values indicated disseminated intravascular coagulation, additional supplementation of eritrocyte suspensions, fresh frozen plasma and trombocyte suspensions were required intra and postoperatively. Eventually the patient was hemodynamically stable at the early postoperative days.

However, after one week, fever $\left(38-39^{\circ} \mathrm{C}\right)$ and abdominal distention were observed. The patient suffered from pelvic pain and gastrointestinal intolerance. Further evaluation by ultrasonography revealed intraabdominal local heterogeneous fluid accumulation and distented bowel loops. Abdominal magnetic resonance imaging confirmed the diagnosis as heterogeneous fluid collection. The clinical symptoms did not improve despite combined extended spectrum antibiotics. Therefore percutaneous drainage of the collection was per- 
formed by a pig-tail catheter through anterior abdominal wall. $1500 \mathrm{cc}$ hemorrhagic dense fluid was discharged. During follow up, clinical findings improved in a few days and the patient was discharged with oral antibiotherapy.

\section{Discussion}

Evenif surgical hemostasis is achieved in uterine atony cases intraoperatively, close follow-up of these cases is required in the early postoperative period. Especially in cases complicated with disseminated intravascular coagulation, pelvic hematoma might occur in short term after surgical management. In cases with consumptive coagulopathy due to massive hemorrhage, an intraabdominally replaced surgical drain will help in postoperative follow-up. By this way, continuing bleeding collected in the abdominal cavity can be recognized in short term, and drainage of the accumulating blood will prevent possible abdominal/peritoneal infections in long term. For cases in whom the drainage is not constituted, intraabdominal collection acompanied by abdominal distention, pain, gastrointestinal symptoms and fever, might result with need for further diagnostic and therapeutic interventions.

Imaging techniques (computed tomography, magnetic resonance imaging) and laboratory tests (white blood cells, C-reactive protein, sedimentation) may be helpful to differentiate between an infected or a non-infected collection in such cases. In symptomatic cases, drainage may be held percutaneously or by laparotomy. If percutaneous drainage is feasible anatomically and technically as in this case presented here, than the collection may be drained by a less invasive procedure with a shorter recovery period. If not, than relaparotomy might be necessiated.

As a result, in order to avoid the risk of any intraabdominal collection intraoperative drain replacement is strongly recommended in surgically managed uterine atony cases especially complicated by consumptive coagulopathy. A 'simple intraoperative intervention' in such cases may avoid short and long term need for further surgical attempts.

\section{Conclusion}

The surgical interventions for uterine atony may constitute of different steps according to the origin of the hemorrhage, preference and skills of the surgeon. ${ }^{2} \mathrm{UCS}$, widely performed as an easy approach, does not have a standard neither for the procedure itself nor for the suture material used. Although UCS are highly effective for preservation of the uterus, a damaged endometrium or totally obliterated uterine cavity in long term may lead to loss of fertility. Therefore, according to the data in the literature B-Lynch suture seems to be better than other UCS techniques for preservation of the fertility in longterm period. ${ }^{18}$ More specific data and analysis are required to clarify the manifestation of long term fertility outcomes after
UCS. Additionally after UCS, there may be a need for recurrent surgical interventions to stabilize the medical status and/or fertility of the patient. After all, hysteroscopic and laparoscopic second look evaluation in such patients may improve adverse outcomes.

\section{References}

1. World Health Organization. WHO Guidelines for the Management of Postpartum Haemorrhage and Retained Placenta 2009 Available from: http://whqlibdoc.who.int/ publications/2009/9789241598514_eng.pdfa

2. Rath W, Hackethal A, Bohlmann MK. Second-line treatment of postpartum haemorrhage (PPH). Arch Gynecol Obstet 2012;286(3):549-61.

3. B-Lynch C, Coker A, Lawal AH, Abu J, Cowen MJ. The B-Lynch surgical technique for the control of massive postpartum hemorrhage: an alternative to hysterectomy? Five cases reported. Br J Obstet Gynaecol 1997;104:372-5.

4. Hackethal A, Brueggmann D, Oehmke F, Tinneberg HR, Zygmunt MT, Muenstedt K. Uterine compression U-sutures in primary postpartum hemorrhage after Cesarean section: fertility preservation with a simple and effective technique. Hum Reprod 2008;23(1):74-9.

5. Matsubara S, Yano H, Ohkuchi A, Kuwata T, Usui R, Suzuki M. Uterine compression sutures for postpartum hemorrhage: an overview. Acta Obstet Gynecol Scand 2013;92(4):378-85.

6. Gizzo S, Saccardi C, Patrelli TS et al. Fertility rate and subsequent pregnancy outcomes after conservative surgical techniques in postpartum hemorrhage: 15 years of literature. Fertil Steril 2013;99(7):2097-107.

7. AbdRabbo SA. Stepwise uterine devascularization: a novel technique for management of uncontrolled postpartum hemorrhage with preservation of the uterus. Am J Obstet Gynecol 1994;171(3):694-700.

8. Amorim-Costa C, Mota R, Rebelo C, Silva PT. Uterine compressionsutures for postpartum hemorrhage: is routine postoperative cavity evaluation needed? Acta Obstet Gynecol Scand 2011;90(7):701-6.

9. Rathat G, Do Trinh P, Mercier G et al. Synechia after uterine compression sutures. Fertil Steril 2011;95(1):405-9.

10. Poujade O, Grossetti A, Mougel L, Ceccaldi PF, Ducarme $\mathrm{G}$, Luton D. Risk of synechiae following uterine compression sutures in the management of major postpartum haemorrhage. BJOG 2011;118(4):433-9.

11. Ochoa M, Allaire AD, Stitely ML. Pyometria after hemostatic square suture technique. Obstet Gynecol 2002; 99(3):506-9.

12. Wu HH, Yeh GP. Uterine cavity synechiae after hemostatic square suturing technique. Obstet Gynecol 2005;105: 1176-8.

13. Dadhwal V, Sumana G, Mittal S. Hematometra following 
uterine compression sutures. Int J Gynaecol Obstet 2007; 99(3):255-6.

14. Friederich L, Roman H, Marpeau L. A dangerous development. Am J Obstet Gynecol 2007;196:92.

15. Treloar EJ, Anderson RS, Andrews HS, Bailey JL. Uterine necrosis following B-Lynch suture for primary postpartum hemorrhage. BJOG 2006;113:486-8.

16. Joshi VM, Shrivastava M. Partial ischemic necrosis of the uterus following a uterine brace compression suture.
BJOG 2004;111:279-80.

17. Somalwar SA, Joshi SA, Bhalerao A, Kawthalkar AS, Jain S, Mahore S. Total Uterine Necrosis: A Complication of B-Lynch Suture. Journal of South Asian Federationof Obstetrics and Gynaecology 2012;4(1):61-3

18. Mallappa Saroja CS, Nankani A, El-Hamamy E. Uterine compression sutures, an update: review of efficacy, safety and complications of B- Lynch suture and other uterine compression techniques for postpartum haemorrhage. Arch Gynecol Obstet 2010;281(4):581-8. 